\section{Avaliação do sistema de pontuação para o diagnóstico da tuberculose na infância preconizado pelo Ministério da Saúde, Brasil}

\author{
Evaluation of a scoring system recommended by \\ the Brazilian Ministry of Health for the diagnosis of \\ childhood tuberculosis
}

\footnotetext{
${ }^{1}$ Núcleo de Doenças Infecciosas, Universidade Federal do Espírito Santo, Vitória, Brasil.

2 Escola Nacional de Saúde Pública Sergio Arouca, Fundação Oswaldo Cruz, Rio de Janeiro, Brasil.

3 Instituto de Medicina Social, Universidade do Estado do Rio de Janeiro, Rio de Janeiro, Brasil.

Correspondência

E. L. N. Maciel Laboratório de Epidemiologia, Núcleo de Doenças Infecciosas, Centro de Ciências da Saúde,

Universidade Federal do Espírito Santo.

Av. Marechal Campos 1468, Vitória, ES

29040-091, Brasil.

emaciel@ndi.ufes.br
}

\begin{abstract}
Tuberculosis is currently one of the main causes of death in the world. One of the difficulties for tuberculosis diagnosis in childhood is the bacteriological identification of Mycobacterium tuberculosis. This nested case-control study aimed to compare the diagnostic values of a scoring system proposed by the Brazilian Ministry of Heath for diagnosis of childhood tuberculosis using gastric washing samples taken in the outpatient and hospital settings. A total of 108 children underwent gastric washing (53 with and 55 without tuberculosis). The scoring system proposed by the Brazilian Ministry of Heath for diagnosis of tuberculosis in children with negative sputum smears showed good sensitivity in both groups, and the best cutoff point was "1" (possible tuberculosis), with 92\% sensitivity, thus feasible for use as an ancillary diagnostic test in children submitted to gastric washing. Our recommendation is that the Ministry of Health scoring system be used by health services to assist the physician in deciding on whether to continue the investigation of childhood tuberculosis, and not only in children who have already undergone the procedure and who have shown negative results.
\end{abstract}

Tuberculosis; Diagnosis; Child
Ethel Leonor Noia Maciel 1

Reynaldo Dietze 1

Rita Elizabeth Checon de Freitas Silva ${ }^{1}$

David Jamil Hadad 1

Claudio Jose Struchiner 2,3

\section{Introdução}

A tuberculose é uma das mais importantes causas de mortalidade em todo o mundo. Cerca de um terço da população mundial está infectada pelo Mycobacterium tuberculosis 1 . Nos países em desenvolvimento estima-se que existam, na faixa etária abaixo de 15 anos, aproximadamente 1.300.000 casos, com ocorrência de 450 mil mortes por tuberculose anualmente 2,3 . No Brasil, estima-se que, do total dos casos notificados, $15 \%$ sejam de tuberculose em menores de 15 anos 4 . No Estado do Espírito Santo, dos 1.620 casos novos notificados no ano de 1999, 104 (6,4\%) eram em menores de 15 anos 5 .

Os sinais e sintomas da tuberculose na infância são inespecíficos, o que dificulta a suspeição clínica e retarda o diagnóstico da doença. $\mathrm{O}$ espectro clínico é extremamente variado e vai desde formas assintomáticas até formas graves disseminadas, com emagrecimento importante e evolução para o óbito ${ }^{6}$. O exame radiológico do tórax, apesar de pouco específico, é um dos pilares para o diagnóstico, análise da evolução das lesões e avaliação da resposta terapêutica 7 . Entretanto, esses achados muitas vezes são insuficientes para justificar o início do tratamento, daí a necessidade de se associar outros parâmetros para a definição do caso 8 .

O teste tuberculínico na criança traz como complicador a interpretação dos resultados em menores de dois anos vacinados com BCG. Ape- 
sar do novo consenso do Ministério da Saúde definir como positiva somente uma reação igual ou superior a $15 \mathrm{~mm}$ nos vacinados há menos de dois anos e superior a $10 \mathrm{~mm}$ naqueles vacinados há mais de dois anos, estas interpretações ainda não são consenso na literatura 9,10,11.

Quando, entretanto, os problemas com a suspeição clínica conseguem ser contornados, surgem os problemas relacionados à confirmação bacteriológica do caso, diagnóstico de certeza da doença, que geralmente constitui o pré-requisito para o início do tratamento em adultos. Nas crianças, os índices baixos de positividade da cultura do escarro ou do lavado gástrico (30\% a $40 \%$ ) estão pautados, além de problemas técnicos, na própria patogênese da doença nesta faixa etária, que é paucibacilar, o que dificulta ainda mais a confirmação bacteriológica 12,13,14,15,16.

Existem atualmente seis sistemas de pontuação para o diagnóstico da tuberculose infantil propostos nos últimos anos por pesquisadores ou órgãos ligados à área da saúde de diversos países 17,18,19,20,21,22. Alguns desses sistemas não são aplicáveis a países como o Brasil, onde a doença é considerada de alta endemicidade e a vacinação com BCG intradérmico faz parte do calendário oficial de vacinação 23 . Outros sistemas foram feitos para países ou regiões onde a infra-estrutura de saúde é precária ou quase inexistente.

Recentemente, o Ministério da Saúde propôs um novo sistema de pontuação para ser utilizado em crianças com suspeição da doença, mas com baciloscopia negativa ${ }^{9}$. Esse sistema é uma compilação dos sistemas anteriores adaptada para a realidade do nosso sistema único de saúde e à situação epidemiológica da doença. Desde o seu lançamento em 2002, esse sistema de pontuação foi avaliado de forma retrospectiva em dois estudos 17,18 . Somente um deles utilizou a cultura como padrão ouro na avaliação do sistema de escore. Neste estudo, 45 (32\%) de 141 crianças que haviam feito lavado gástrico tiveram o diagnóstico confirmado por cultura, e em 96 (68\%) o diagnóstico da doença foi descartado 17 . A sensibilidade e especificidade do sistema de pontuação do Ministério da Saúde foi respectivamente $88,9 \%$ e $86,5 \%$. O nosso estudo teve por objetivo avaliar o sistema de pontuação do Ministério da Saúde para o diagnóstico de tuberculose em crianças em um estudo casocontrole aninhado em uma coorte de pacientes que haviam sido arrolados para participar de um estudo comparativo entre a coleta ambulatorial e hospitalar do lavado gástrico no diagnóstico da doença.

\section{Sujeitos e métodos}

\section{Descrição do estudo}

Desenho híbrido caso-controle aninhado em uma coorte prospectiva para avaliação do sistema de pontuação do Ministério da Saúde, em crianças com suspeita clínica de tuberculose. Neste estudo além do lavado gástrico utilizouse como critério diagnóstico da doença a notificação do caso e encerramento do mesmo sem mudança de diagnóstico.

\section{Coleta de dados}

A coorte prospectiva tinha por objetivo comparar a sensibilidade e especificidade de duas formas de coleta de lavado gástrico, e será descrita a seguir. Os pacientes com indicação para realização de lavado gástrico foram arrolados no período de julho de 1999 a agosto de 2003, em dois hospitais do Município de Vitória, Espírito Santo: o Hospital Universitário Cassiano Antonio Moraes (HUCAM) e o Hospital Infantil Nossa Senhora da Glória (HINSG). A internação em ambos os hospitais era feita a partir de uma central de vagas que administra todas as internações no Estado do Espírito Santo desde 1999. O critério de escolha do hospital baseava-se somente na disponibilidade de vagas em cada um deles. As coletas de lavado gástrico variaram conforme o local do estudo: no HUCAM, a coleta foi ambulatorial e no HINSG, com internação hospitalar 23.

Durante o período do estudo foram realizados lavados gástricos em 221 crianças. Desse total, 53 tiveram o diagnóstico de tuberculose: em 32 a confirmação foi bacteriológica e em 21 o diagnóstico baseou-se em sinais clínicos e/ou radiológicos. No restante, 168 crianças, o diagnóstico da tuberculose foi descartado. Dessa coorte foram retirados os 53 pacientes que constituíram o grupo caso do presente estudo. Os 55 pacientes do grupo controle foram selecionados do "pool" de 168 crianças nas quais o diagnóstico de tuberculose foi descartado. Elas foram escolhidas com base em uma tabela de números aleatórios gerada por computador. Todas as crianças tinham sorologia negativa para o HIV.

\section{Procedimentos do estudo}

Construiu-se uma ficha de coleta de dados que continha todos os itens do sistema de pontuação do Ministério da Saúde (Tabela 1). As fichas eram preenchidas com os dados do paciente e entregues a dois pesquisadores médicos que classificavam as crianças em uma das três categorias: diagnóstico pouco provável (critério 0), diag- 


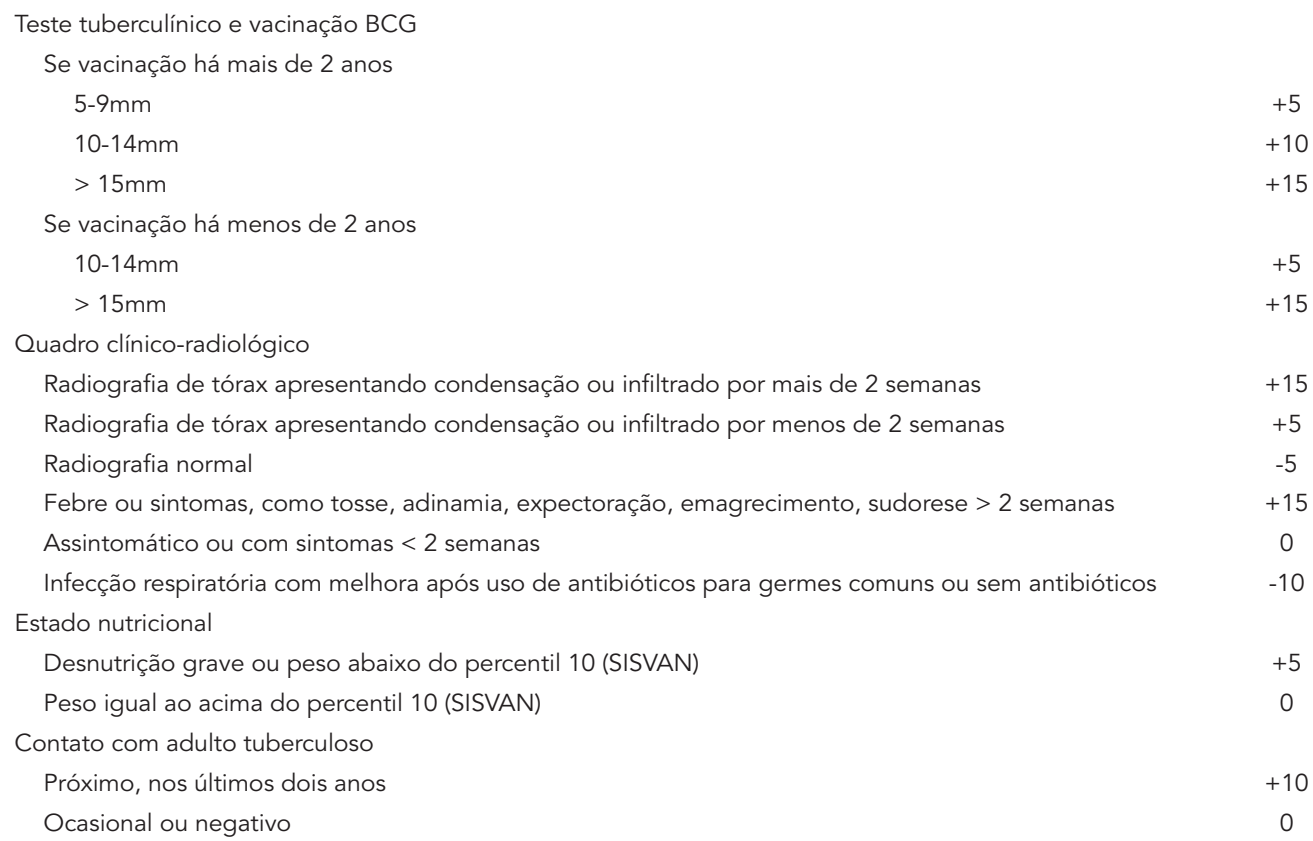

Interpretação: $\geq 40$ pontos (diagnóstico muito provável); 30-35 pontos (diagnóstico possível); $\leq 25$ pontos (diagnóstico pouco provável).

nóstico possível (critério 1), diagnóstico muito provável (critério 2). Os pesquisadores faziam a classificação de forma independente e cega para o grupo de estudo. Em caso de classificação discordante o desempate era feito por um terceiro pesquisador.

O padrão ouro utilizado foi o diagnóstico médico e/ou bacteriológico com notificação do caso e seu encerramento sem mudança diagnóstica.

\section{Análise estatística}

Na avaliação e validação do sistema de pontuação do Ministério da Saúde, calculou-se o ponto de corte dos critérios de pontuação para o diagnóstico da tuberculose por meio da sensibilidade, especificidade com seus respectivos intervalos de confiança para caso e controles e, também o índice de kappa na avaliação da concordância entre os pesquisadores. A análise das médias de idade foi feita pelo teste $\mathrm{t}$ de Student e para as demais variáveis dicotômicas foi calculado o odds ratio, ambos os testes bicaudais com um nível de significância de 0,05, utilizando-se o pacote Stata 8.0 (Stata Corp., College Station, Estados Unidos).

\section{Aspectos éticos}

Este estudo foi aprovado pelo Comitê de Ética em Pesquisa do Centro Biomédico da Universidade Federal do Espírito Santo (no. 023/99).

\section{Resultados}

Das 53 crianças diagnosticadas como casos, 23 realizaram o lavado gástrico em nível ambulatorial e 30, hospitalar. Das 55 crianças consideradas controles, 31 realizaram o lavado gástrico ambulatorial e 24, hospitalar (Tabela 2). A classificação das crianças de acordo com os critérios do Ministério da Saúde revelou um bom nível de concordância entre os pesquisadores [índice de kappa: 0,92 (IC: 0,78-0,96)].

Não houve diferenças sócio-demográficas entre os dois grupos de pacientes em relação ao aos casos e controles (Tabela 3). A média de idade dos pacientes também foi semelhante: 43,4 meses (amplitude 8-168 meses) no grupo caso e 42,6 meses (amplitude 1-144 meses) no grupo controle (test t de Student 0,077; $\mathrm{p}=0,53$ ). 
Distribuição do sistema pontuação do Ministério da Saúde de acordo com os grupos de estudo e o diagnóstico de tuberculose.

\begin{tabular}{|c|c|c|c|c|c|c|c|c|}
\hline \multirow[t]{4}{*}{ Variáveis } & \multicolumn{8}{|c|}{ Local de coleta do lavado gástrico } \\
\hline & \multicolumn{4}{|c|}{ Tuberculose positivo (casos) } & \multicolumn{4}{|c|}{ Tuberculose negativo (controles) } \\
\hline & \multicolumn{2}{|c|}{$\begin{array}{l}\text { Ambulatorial } \\
(n=230)\end{array}$} & \multicolumn{2}{|c|}{$\begin{array}{l}\text { Hospitalar } \\
\qquad(n=30)\end{array}$} & \multicolumn{2}{|c|}{$\begin{array}{l}\text { Ambulatorial } \\
\qquad(n=31)\end{array}$} & \multicolumn{2}{|c|}{$\begin{array}{l}\text { Hospitalar } \\
\qquad(n=24)\end{array}$} \\
\hline & $f$ & $\%$ & f & $\%$ & $f$ & $\%$ & $f$ & $\%$ \\
\hline Critério 0 & 0 & 0,0 & 4 & 13,0 & 22 & 71,0 & 16 & 66,0 \\
\hline Critério 1 & 12 & 52,0 & 9 & 30,0 & 16 & 16,0 & 4 & 17,0 \\
\hline Critério 2 & 11 & 48,0 & 17 & 57,0 & 4 & 13,0 & 4 & 17,0 \\
\hline
\end{tabular}

Tabela 3

Distribuição das variáveis sócio-demográficas de acordo com o diagnostico da doença.

\begin{tabular}{|c|c|c|c|c|c|c|c|c|c|}
\hline \multirow[t]{3}{*}{ Variáveis } & \multicolumn{4}{|c|}{ Casos } & \multicolumn{4}{|c|}{ Controles } & \multirow[t]{3}{*}{ OR (IC) } \\
\hline & \multicolumn{2}{|c|}{$\begin{array}{l}\text { Ambulatorial } \\
\qquad(n=23)\end{array}$} & \multicolumn{2}{|c|}{$\begin{array}{l}\text { Hospitalar } \\
(n=30)\end{array}$} & \multicolumn{2}{|c|}{$\begin{array}{l}\text { Ambulatorial } \\
\qquad(\mathrm{n}=31)\end{array}$} & \multicolumn{2}{|c|}{$\begin{array}{l}\text { Hospitalar } \\
(n=24)\end{array}$} & \\
\hline & $f$ & $\%$ & $f$ & $\%$ & $f$ & $\%$ & $f$ & $\%$ & \\
\hline Sexo & & & & & & & & & $1,15(0,49-2,69)$ \\
\hline Masculino & 7 & 30,4 & 14 & 46,6 & 10 & 32,2 & 10 & 41,6 & \\
\hline Feminino & 16 & 69,6 & 16 & 53,4 & 21 & 68,8 & 14 & 58,4 & \\
\hline Procedência & & & & & & & & & $1,13(0,46-2,81)$ \\
\hline Grande Vitória & 18 & 78,0 & 20 & 66,6 & 22 & 71,0 & 16 & 66,6 & \\
\hline Interior do Estado & & & & & & & & & \\
\hline do Espírito Santo & 5 & 22,0 & 10 & 33,4 & 9 & 29,0 & 8 & 33,4 & \\
\hline Idade (anos) & & & & & & & & & $1,22(0,42-3,61)$ \\
\hline$\leq 5$ & 18 & 78,0 & 26 & 86,6 & 24 & 77,0 & 20 & 83,3 & \\
\hline $6-14$ & 5 & 22,0 & 4 & 13,4 & 7 & 23,0 & 4 & 16,7 & \\
\hline
\end{tabular}

O melhor desempenho diagnóstico ocorreu quando se utilizou como ponto de corte os critérios 1 e 2 como definição de tuberculose (Tabela 4 ). No corte $\geq 30$ pontos (critério 1 ) a sensibilidade foi $92 \%$ (IC: $86-99$ ). No corte $\geq 40$ pontos (critério 2) a sensibilidade foi $40 \%$ (IC: 29-67).

Como forma de validar com critérios mais rígidos o sistema de pontuação do Ministério da Saúde, fizemos uma análise estratificada da sensibilidade utilizando como padrão ouro a confirmação microbiológica dos casos e ponto de corte $\geq 30$ pontos. Com esses critérios, a amostra do grupo caso foi composta por 33 pacientes (15 oriundos do grupo ambulatorial e 18 do grupo hospitalar) 24 . Nessas condições a sensibilidade do sistema de pontuação foi de $96 \%$ (IC: 88-99), 4 pontos porcentuais acima da sensibilidade obtida na análise original.

\section{Discussão}

No Estado do Espírito Santo, a maior concentração de profissionais especializados em tuberculose situa-se na capital, fato que justifica a representatividade de nossa amostra. Validações de sistemas de pontuação em doenças de difícil diagnóstico, que sejam de fácil operacionalização e baixo custo, devem ser incentivadas.

Desde a sua recomendação pelo Ministério da Saúde, o Sistema de Pontuação para Diagnóstico de Crianças Negativas à Baciloscopia para Tuberculose foi avaliado em somente dois estudos retrospectivos realizados por Sant'Anna et al. 16,17. Nesses estudos houve uma elevada associação entre os resultados da pontuação e os diagnósticos presentes nos prontuários dos pacientes. O melhor ponto de corte encontrado foi 
Distribuição de casos e controles em relação ao ponto de corte nos critérios 1 e 2 .

\begin{tabular}{|c|c|c|c|c|c|}
\hline \multirow[t]{2}{*}{ Pontos de corte } & \multicolumn{3}{|c|}{ Diagnóstico de TB } & \multirow{2}{*}{$\begin{array}{c}\text { Sensibilidade } \\
\text { (IC95\%) }\end{array}$} & \multirow{2}{*}{$\begin{array}{c}\text { Especificidade } \\
\text { (IC95\%) }\end{array}$} \\
\hline & Positivo & Negativo & Total & & \\
\hline \multicolumn{6}{|l|}{ Critério 1} \\
\hline$\geq 30$ pontos & 49 & 17 & 66 & & \\
\hline$\leq 25$ pontos & 4 & 38 & 42 & $92 \%(86-99)$ & $70 \%(54-84)$ \\
\hline Total & 53 & 55 & 108 & & \\
\hline \multicolumn{6}{|l|}{ Critério 2} \\
\hline$\geq 40$ pontos & 21 & 15 & 36 & & \\
\hline$\leq 30$ pontos & 32 & 40 & 72 & $40 \%(29-67)$ & $85 \%(71-95)$ \\
\hline Total & 53 & 55 & 108 & & \\
\hline
\end{tabular}

o de tuberculose possível com 30 pontos (sensibilidade de $88,9 \%$ e especificidade de $86,5 \%$ ). Os dados do nosso estudo foram semelhantes (sensibilidade de $92 \%$ e especificidade de $70 \%$ ) e reforçam os resultados dos estudos de Sant'Anna et al. 16,17. Não houve diferença entre o desempenho dos pontos de corte do nosso estudo em relação ao local de realização do lavado gástrico 23 . Esse resultado valida a utilização desse sistema de pontuação tanto no nível ambulatorial quanto hospitalar. Vale ressaltar que quando utilizamos o padrão ouro ideal (confirmação microbiológica dos casos) na definição dos pacientes do grupo caso, houve um incremento de apenas $4 \%$ na sensibilidade do sistema de pontuação, o que reforça o bom desempenho do sistema em condições em que a cultura não pode ser realizada. Essa diferença pode ainda ser explicada pelo fato das crianças que apresentaram cultura positiva terem apresentado sintomas mais exuberantes da doença. Destaca-se que esses são os desafios encontrados na tomada de decisão na assistência em crianças com suspeita de tuberculose visto que, amostras coletadas por meio de lavado gástrico dificilmente são positivas à baciloscopia 14 e um resultado de cultura demora em média 60 dias para ser finalizado. Reforça esse dado o fato de que em nosso estudo a tomada de decisão acerca do início do tratamento esteve ligada ao resultado da cultura em somente dois pacientes.

O sistema de pontuação proposto pelo Ministério da Saúde apresenta boa sensibilidade. Usualmente testes com alta sensibilidade são os de escolha quando não se pode correr o risco de não se diagnosticar uma doença potencialmente grave se não tratada, como a tuberculose 24 .
Os testes sensíveis também são importantes nos estágios iniciais de um processo diagnóstico, quando um grande número de possibilidades está sendo considerado e se quer reduzi-lo como no caso do instrumento analisado. Ressalta-se ainda que, sua utilização poderia garantir a interrupção ou a necessidade de prosseguimento na investigação diagnóstica, como é o caso do lavado gástrico. Além do mais, se esse instrumento houvesse sido utilizado como teste de rastreamento, mais de $50 \%$ das crianças que foram submetidas ao lavado gástrico no Estado do Espírito Santo teriam sua indicação questionada.

Diante do exposto, conclui-se que a caracterização de sinais e sintomas cujo somatório se encontra entre 30 e 35 pontos do instrumento proposto pelo Ministério da Saúde já seria suficiente para indicar a probabilidade da doença. Outra vantagem que se pode apontar é a explicitação e a homogeneidade na definição dos critérios diagnósticos desse instrumento, o que possibilitaria ao clínico decidir sobre o encaminhamento que melhor se aplicaria àquele paciente, uma vez que, uma pontuação inferior a 30 pontos excluiria a tuberculose dos possíveis diagnósticos diferenciais. Além disso, essa homogeneidade na conduta diagnóstica nos permitiria o estabelecimento de coortes para a avaliação desses resultados em diferentes serviços de atendimento a crianças suspeitas de tuberculose.

Diante do exposto, recomendamos uma implementação de forma mais abrangente no país do sistema de pontuação proposto pelo Ministério da Saúde, não apenas em crianças e adolescentes negativos ao exame de baciloscopia, mas também naqueles em que a tuberculose figure como hipótese diagnóstica. 


\section{Resumo}

A tuberculose é conhecida como uma das mais importantes causas de mortalidade no mundo. O diagnóstico da tuberculose na infância tem entre suas muitas dificuldades o achado bacteriológico do Mycobacterium tuberculosis. Este foi um estudo de caso-controle aninhado em uma coorte prospectiva com crianças que coletaram lavado gástrico em nível ambulatorial e hospitalar, com o objetivo de identificar se o sistema de pontuação para o diagnóstico de tuberculose em crianças negativas à baciloscopia, proposto pelo Ministério da Saúde, poderia ser utilizado como instrumento inicial de identificação do diagnóstico da tuberculose na infância. Um total de 108 crianças foi submetido ao lavado gástrico, sendo que 53 tiveram diagnóstico de tuberculose e 55 com diagnóstico descartado. O sistema de pontuação proposto pelo Ministério da Saúde para o diagnóstico de tuberculose em crianças com baciloscopia negativa apresentou uma boa sensibilidade em ambos os grupos estudados, sendo o melhor ponto de corte o critério 1 (possível diagnóstico de tuberculose) com sensibilidade de $92 \%$ podendo, então, ser usado como um auxiliar na investigação diagnóstica de crianças que se submeterão ao lavado gástrico. Nossa recomendação é que o sistema de pontuação do Ministério da Saúde seja utilizado nos serviços de saúde para auxiliar o médico a decidir sobre continuar ou não a investigação da tuberculose infantil, e não somente em crianças que já foram submetidas ao procedimento e tiveram suas amostras com resultado negativo.

Tuberculose; Diagnóstico; Criança

\section{Colaboradores}

E. L. N. Maciel elaborou a metodologia, analisou os dados e elaborou o manuscrito. R. E. C. F. Silva e D. J. Hadad examinaram e classificaram as crianças de acordo com o sistema de pontuação e também auxiliaram na elaboração do manuscrito. R. Dietze e C. J. Struchiner participaram na elaboração da metodologia, análise e elaboração do manuscrito.

\section{Referência}

1. Frieden TR, Sterling TR, Munsiff SS, Watt CJ, Dye C. Tuberculosis. Lancet 2003; 362:887-99.

2. Starke JR, Jacobs RF, Jereb J. Resurgence of tuberculosis in children. J Pediatr 1992; 120:839-55.

3. Starke JR. Childhood tuberculosis. A diagnostic dilemma [Editorial]. Chest 1993; 104:329-30.

4. Clemax C, Sant'Anna CC, Mourgues LV, Ferrero F, Balanzat AM. Diagnóstico e terapêutica da tuberculose infantil - uma visão atualizada de um antigo problema. J Pediatr (Rio J) 2002; 78:205-14.

5. Maciel ELN, Marinato CA, Bandeira CFR, Tonini MS, Dietze R, Ramos MC. O perfil epidemiológico da tuberculose em crianças e adolescentes menores de 15 anos na Grande Vitória, Brasil, no período de 1990 a 2001. Cad Saúde Colet (Rio J) 2006; 14:81-94.
6. Houwert KA, Borggreven PA, Schaaf HS, Nel E, Donald PR, Stolk J. Prospective evaluation of World Heath Organization criteria to assist diagnosis of tuberculosis in children. Eur Respir J 1998; 11:1116-20.

7. Farhart CK, Carvalho ES, Carvalho LHFR. Infectologia pediátrica. 2a Ed. São Paulo: Editora Atheneu; 1999.

8. Marcondes E, Vaz FAC, Ramos JLA, Okay Y. Pediatria básica. 8a Ed. São Paulo: Editora Sarvier; 2003.

9. Ministério da Saúde. Controle de tuberculose: uma proposta de integração ensino-serviço. Rio de Janeiro: Centro de Referência Prof. Hélio Fraga/ Sociedade Brasileira de Pneumologia e Tisiologia; 2002. 
10. Ministério da Saúde. Normas técnicas para o Programa de Controle da Tuberculose. Brasília: Ministério da Saúde; 2000

11. Laven G. Diagnosis of tuberculosis in children using fluorescence microscopic examination of gastric washings. Am Rev Respir Dis 1977; 115:743-9.

12. Migliori GB, Borghesi A, Rossanigo P, Adriko C, Neri M, Santini S, et al. Proposal of an improved scores method for the diagnosis of pulmonary tuberculosis in childhood in developing countries. Tuber Lung Dis 1992; 73:145-9.

13. Somu N, Swaminathan S, Paramasivan CN, Vijayasekaran D, Chandrabhooshanam A, Vijayan VK, et al. Value of bronchoalveolar lavage and gastric lavage in the diagnosis of pulmonary tuberculosis in children. Tuber Lung Dis 1995; 76:295-9.

14. Donald PR. Childhood tuberculosis. Curr Opin Pulm Med 2000; 6:187-92.

15. Ministério da Saúde. Manual de normas para o controle da tuberculose. Brasília: Coordenação de Pneumologia Sanitária, Centro Nacional de Epidemiologia, Ministério da Saúde; 1995.

16. Sant'Anna CC, Orfalias CTS, March MFBP. A retrospective evaluation of a score system adopted by the Ministry of Health, Brazil, in the diagnosis of pulmonary tuberculosis in childhood: a case control study. Rev Inst Med Trop São Paulo 2003; 45:103-5.

17. Sant'Anna CC, Santos MARC, Franco R. Diagnosis of pulmonary tuberculosis by score system in children and adolescents: a trial in a reference center in Bahia, Brazil. Braz J Infect Dis 2004; 8:305-10.
18. Nair PM, Philip E. A scoring system for the diagnosis of tuberculosis in children. Indian Pediatr 1981; 18:299-303.

19. Migliori GB, Borghesi A, Rossanigo P, Adriko C, Neri M, Santini S, et al. Proposal of an improved scores method for the diagnosis of pulmonary tuberculosis in childhood in developing countries. Tuber Lung Dis 1992; 73:145-9.

20. Fourie PB, Becker PJ, Festenstein F, Migliori GB, Alcaide J, Antunes M, et al. Procedures for developing a simple scoring method based on unsophisticated criteria for screening children for tuberculosis. Int J Tuberc Lung Dis 1998; 2:116-23.

21. Stegen G, Jones K, Kaplan P. Criteria for guidance in the diagnosis of tuberculosis. Pediatrics 1969; 49:260-3.

22. Tidjani O, Amedome A, Ten Dam HG. The protective effect of BCG vaccination of the newborn against childhood tuberculosis in Africa community. Tubercle 1986; 67:269-81.

23. Maciel ELN. Avaliação do diagnóstico da tuberculose na infância [Tese de Doutorado]. Rio de Janeiro: Instituto de Medicina Social, Universidade do Estado do Rio de Janeiro; 2004.

24. Fletcher RH, Fletcher SW, Wagner EH. Clinical epidemiology: the essentials. 3rd Ed. Baltimore: Williams \& Wilkins; 1996

Recebido em 31/Out/2005

Versão final reapresentada em 30/Mai/2007

Aprovado em 16/Ago/2007 